\title{
Influence of Attention Alternation on Movement-Related Cortical Potentials in Healthy Individuals and Stroke Patients
}

\author{
Susan Aliakbaryhosseinabadi ${ }^{\mathbf{a}}$, Vladimir Kostic ${ }^{\mathbf{b}}$, Aleksandra Pavlovic ${ }^{\mathbf{b}}$, Sasa Radovanovic , $^{\mathrm{b}}$, \\ Ernest Nlandu Kamavuako ${ }^{\mathrm{a}}$, Ning Jiang ${ }^{\mathrm{c}}$, Laura Petrini ${ }^{\mathrm{a}, \mathrm{d}}$, Kim Dremstrup ${ }^{\mathrm{a}}$, Dario Farina ${ }^{\mathrm{e}}$ and \\ Natalie Mrachacz-Kersting ${ }^{\text {a }}$
}

${ }^{a}$ Center for Sensory-Motor Interaction (SMI), Department of Health Science and Technology, Aalborg University, Aalborg, Denmark

${ }^{b}$ Neurology Clinic, Clinical Center of Serbia, Faculty of Medicine, University of Belgrade, Dr Subotica 6, Belgrade, Serbia

${ }^{c}$ Department of Systems Design Engineering, Faculty of Engineering, University of Waterloo, Waterloo, Canada

${ }^{\mathrm{d}}$ Department of Communication and Psychology, Center for Cognitive Neuroscience (CCN), Aalborg University, Aalborg, Denmark

e Department of Bioengineering, Imperial College London, SW7 2AZ London, UK

emails: $\quad \underline{\text { sal@hst.aau.dk, }}$ Vladimir.s.kostic@gmail.com, aleksandra3003@yahoo.com, sasar@imi.bg.ac.rs, enk@hst.aau.dk, ning.jiang@uwaterloo.ca, lap@hst.aau.dk, kdn@hst.aau.dk, d.farina@imperial.ac.uk , nm@hst.aau.dk

\section{Corresponding author}

Natalie Mrachacz-Kersting

Center for Sensory-Motor Interaction (SMI), Department of Health Science and Technology, Aalborg University, Aalborg, Denmark.

Email: $\underline{n m @ h s t . a a u . d k}$ 


\begin{abstract}
Objective: In this study, we analyzed the influence of artificially imposed attention variations using the auditory oddball paradigm on the cortical activity associated to motor preparation/execution.

Methods: EEG signals from $\mathrm{Cz}$ and its surrounding channels were recorded during three sets of ankle dorsiflexion movements. Each set was interspersed with either a complex or a simple auditory oddball task for healthy participants and a complex auditory oddball task for stroke patients.

Results: The amplitude of the movement-related cortical potentials (MRCPs) decreased with the complex oddball paradigm, while MRCP variability increased. Both oddball paradigms increased the detection latency significantly $(\mathrm{p}<0.05)$ and the complex paradigm decreased the true positive rate $(T P R)(p=0.04)$. In patients, the negativity of the MRCP decreased while pre-phase variability increased, and the detection latency and accuracy deteriorated with attention diversion.

Conclusion: Attention diversion has a significant influence on MRCP features and detection parameters, although these changes were counteracted by the application of the laplacian method.

Significance: Brain-computer interfaces for neuromodulation that use the MRCP as the control signal are robust to changes in attention. However, attention must be monitored since it plays a key role in plasticity induction. Here we demonstrate that this can be achieved using the single channel $\mathrm{Cz}$.
\end{abstract}

Keywords: Attention, movement-related cortical potential, brain-computer interface, movement detection

Conflict of interest statement: None of the authors have potential conflicts of interest to be disclosed.

Acknowledgements: This study was supported by grants from Det Obelske Familiefond. 


\section{Introduction}

Brain computer interface (BCI) systems translate patterns of brain activity to provide an artificial communication and control channel between the brain and the external environment without using peripheral nerves or muscles. Event-related synchronization/desynchronization, readiness potentials and movement-related cortical potentials (MRCP) extracted from the time or frequency domain of the electroencephalogram (EEG) are just some examples of signals that have been successfully implemented within BCIs (Jiang et al., 2015; Xu et al., 2014; Xu et al., 2016). To date most BCI studies have been performed in controlled experimental conditions to reduce the effect of noise and distractors. However, real-life scenarios are more complex environments where it is not possible to control the user's status, such as attention or fatigue (Shenoy et al., 2006; Zander and Jatvez, 2012).

In such an environment, the $\mathrm{BCI}$ user will constantly be exposed to various types of sensory stimuli arising both from within the body or the surrounding environment. Attention is a filtering mechanism that allows humans and animals to select only relevant stimuli (Esghaei and Daliri, 2014; Treder et al., 2014) and if directed towards a particular sensory event can modulate brain signals (Treder et al., 2014). It is also considered as a gateway to learning and memory since we typically learn and remember more about stimuli in the environment that we attend to than about stimuli we ignore (Desimone, 1996). Two common types of attention that may affect a BCI user's performance are alternative and divided attention where attention is either shifted between tasks or divided between two or more tasks. In BCIs designed for neurorehabilitation, fast and reliable detection of movement intention is of central importance as this provides the trigger for the accurately timed control of the rehabilitation device (Koyas et al., 2013). If the subject's attention is diverted from the main task to be trained, the detection of intention may have lower accuracy (Albares et al., 2011; Kimura et al., 2008). However, currently little is known regarding the effect that the attention of the user has on brain signal parameters commonly used in BCIs.

Our group has developed a BCI for neuromodulation based on detection of movement intention from specific features of the MRCP. The MRCP is a slow cortical potential that has been associated with voluntarily executed, self-paced or imagined movements (Hallett, 1994). By pairing the intent of the participant with the artificial production of the imagined or intended movement, we have shown significant plastic changes within the motor cortex of both healthy participants and patients (Scheel et al., 2015; Mrachacz-Kersting et al., 2015). Patients also significantly increased their $10 \mathrm{~m}$ walking speed and foot tapping frequency. While these initial results are

promising, in order to use such a BCI system in the daily clinic, a BCI system must be sufficiently robust such that changes in the mental state of the user by environmental factors, such as attention, task learning and fatigue (Li et al., 2012; Roy et al., 2013; Toppi et al., 2014) do not affect the BCIs performance. 
The aim of this study was to investigate the effect of switching the user's attention between two different tasks on the offline performance of our detection algorithm based on MRCPs. This is the first step towards a design for an adaptable detection algorithm that can capture the changes in attention of the user. The results will have important implications for the design of a BCI to be used in real life settings, since cortical plasticity induction is known to be affected by attention on the task (Stefan et al., 2004; Ziemann et al., 2008). We hypothesized that a decrease in attention due to an attention switch between two tasks would directly influence the MRCP characteristics in healthy participants as well as chronic stroke patients, and therefore the BCI performance. 


\section{Materials and Methods}

Two experiments were conducted to quantify the effect of attention in healthy participants and chronic stroke patients. In the first experiment, 18 healthy participants were included and attention effects on MRCP parameters were measured by using two levels of task complexity. In the second experiment, the task complexity that was demonstrated to have the greatest effects on attention diversion in experiment 1 was used on seven stroke patients to investigate how patients' performance was affected.

\subsection{Experiment 1}

\subsubsection{Participants}

Twenty right-handed participants (six females, twelve males) aged 20 to 32 years (mean age 24.33 years) with normal hearing and with no history of neurological disease took part in this study. Two of them, one from each group, was excluded because a lot of artifacts were contaminated. The procedure was approved by the local ethical committee for the region Northern Jutland (N20130039), and all participants signed a written consent form.

\subsubsection{Experimental set-up}

Ten channels of monopolar EEG were recorded using an active EEG electrode system (g. GAMMAcap ${ }^{2}$, Austria) and g.USBamp amplifier (gTec, GmbH, Austria) from FP1, Fz, FC1, FC2, C3, Cz, C4, CP1, CP2, and $\mathrm{Pz}$ according to the standard international 10-20 system. The channel selection was based on the large Laplacian with $\mathrm{Cz}$ as the central channel (McFarland et al., 1997). The reference electrode was placed on Fz and the ground on the left earlobe. A single channel surface electromyography (EMG) was recorded from the tibialis anterior (TA) muscle to control for the subject's movement. All signals were sampled at a frequency of $256 \mathrm{~Hz}$ (16 bits accuracy) and hardware filtered from 0 to $100 \mathrm{~Hz}$.

\subsubsection{Movement and auditory oddball tasks}

Each participant was seated in a comfortable chair while both the right and left leg were resting on a step with the knee and ankle joint flexed $90^{\circ}$. A digital computer screen was placed approximately one meter in front of the participant to show the visual paradigm. Conventional headphones were used to play the auditory stimuli for parts of the experiment. A diagram of the system configuration is presented in figure 1a. Each experiment consisted of two separate blocks with specific repetitions of either the movement or oddball task, described in detail below and also shown in figure 2 . 
The movement task: A visual cue comprised of five phases defined as focus, preparation, execution, hold and rest time (figure 1b) was provided to the participants. After a random duration of focus time, the drawing of a ramp appeared on the screen. A cursor moved along the ramp and when it reached the upward turn, the movement period commenced and participants had to perform and sustain an ankle dorsiflexion for $2 \mathrm{~s}$. The hold phase was followed by a rest period with a random duration of 3-5 s. Participants completed three sets of 30 dorsiflexion trials.

The oddball task: The healthy participants were divided into two groups of nine participants that had to perform one of two designed auditory oddball tasks. In the simple auditory oddball task, a frequent $500 \mathrm{~Hz}$ tone, which is referred to as the standard tone, was randomized with a probability of $80 \%$ with a rare $1200 \mathrm{~Hz}$ tone referred to as the target tone with the probability of $20 \%$. In the complex auditory oddball task, target and standard tone were combined with an additional $1900 \mathrm{~Hz}$ tone (deviate tone). The probability of occurrence of the standard tone was $60 \%$ while target and deviate tones each had a probability of $20 \%$. All stimuli had the same loudness of $75 \mathrm{~dB}$ sound pressure level (SPL) with $200 \mathrm{~ms}$ duration, a $5 \mathrm{~ms}$ rise/fall time and a randomized inter-stimulus interval (ISI) of 1.5-2.5 sec. Thirty target tones were presented among 150 repetitions of tones and participants had to respond to the target tones by pressing a button with their right index finger.

Each participant completed two experimental conditions as presented in figure 2 where the movement and oddball tasks were varied as outlined below:

Control condition: participants in both groups were instructed to do a dorsiflexion according to the visual cue described above, for a total of three sets, each of which included 30 trials of dorsiflexion and was separated from the other set with a minimum of four minutes of rest time.

Low attention condition: In the first group, 30 trials of dorsiflexion in each of three separate sets of the visual paradigm were recorded as for the control experiment. In addition, the low attention auditory oddball task, which included standard and target tones, was presented between sets of dorsiflexion movements.

High attention condition: In the second group, 30 trials of dorsiflexion in each of three separate sets of the visual paradigm were recorded as for the control experiment. In addition, the high attention auditory oddball task, which included standard, target and deviate tones, was presented between sets of dorsiflexion movements.

\subsubsection{ERP analysis}

EEG signals were filtered with a $2^{\text {nd }}$ order Butterworth filter in the range of 3-30 Hz and then divided to single trials from [- $-0.10 .7]$ s based on the start time of each of the oddball tones. The P300 was defined as the maximum value in this time interval and was compared between the simple and complex oddball paradigms to quantify the level of attention. It was expected that the lower attention condition resulted in a lower amplitude 
and an increased P300 latency as compared to the high attention task (Bentin and McCarthy, 1994; Wang et al., 2014).

\subsubsection{MRCP analysis}

EEG signals were band pass-filtered in the frequency range of $0.05-3 \mathrm{~Hz}$ with a $2^{\text {nd }}$ order Butterworth filter and the DC component was removed by subtracting signals from their mean values. In our previous studies we have demonstrated that $\mathrm{Cz}$ is the superior channel for detection (Aliakbaryhosseinabadi et al., 2014) of simple dorsiflexion, so $\mathrm{Cz}$ was used as the channel for movement analysis (Aliakbaryhosseinabadi et al., 2014; Xu et al., 2014). In addition, the large Laplacian of $\mathrm{Cz}$ was calculated with a linear combination of surrounding channels where each channel has a fixed coefficient as indicated in equation 1:

$$
c_{i}=\left\{\begin{array}{cl}
1, & i=1 \\
-\frac{1}{\left(N_{c h}-1\right)} & i \neq 1
\end{array}\right.
$$

$\mathrm{N}_{\mathrm{ch}}$ indicates the number of channels used in large Laplacian ( 9 in the current study).

Trials contaminated by EOG artifacts ( $100 \mu \mathrm{V}$ as a threshold) were removed off-line and epochs extracted from three seconds prior to the cue presentation until three seconds after the cue. All offline analysis was done on a PC using MATLAB (R2014b).

\subsubsection{MRCP features}

Nine temporal features were extracted from the EEG trials as illustrated in figure 1c. Value and time of peak negativity (VPN and TPN), MRCP variability which was defined as the standard deviation among single trials and the MRCP slopes were extracted in three temporal sections: [-2 1] s, [-1 0] s and [ -2 0] s prior to TPN. In addition, the Rebound Rate (RR), computed as the slope between TPN and $1 \mathrm{~s}$ after this point, was used as another feature (Gu et al., 2013). Among these features, RR was from the re-afferent part of the EEG signals and related to post-movement brain activity, while the other features were extracted from the initial negative part of the signals and represent pre-movement brain activity.

The other properties used to compare the effect of alterations in attention were movement detection latency and corresponding true positive rate (TPR) defined as the number of true detections divided by the total number of true events and false positive rate (FPR) defined as the number of false detections divided by the number of total events. The Locality preserving projection followed by a LDA classifier (LPP-LDA) was used to detect the movement intention. The details of this method are presented in (Xu et al., 2014). In this detection technique, the 
signal and noise part of continues EEG signals are extracted based on movement onset obtained from EMG signals. These are subsequently projected into a new feature space with lower dimension by applying the LPP method. Finally, they are classified into two groups within the signal and noise section. If two consecutive classification output represent signal class, a detection occurred. Nine extracted temporal features and three detection parameters (DL, TPR, FPR) were compared within each group of attention task complexity.

\subsubsection{Statistics}

ERP components between two attention levels were compared using independent paired t-tests and one-way ANOVA was used within each task condition to quantify the effect of 'Set' with three levels. To determine the effect of the two attention tasks on the MRCP, two-way nested analysis of variance (ANOVA) was used where participants were nested within difficulty. 'Set' with three levels and 'Attention statuses' with two levels (control and shifted attention) was used as fixed factor. Bonferroni post-hoc test was used in multi pairwise comparison. In addition, one-way ANOVA with three levels of 'Set' was applied within each class of attention levels (control or shifted attention) to find out the differences among three sets. The results were assumed significant if $\mathrm{p} \leq 0.05$. The normality of the data was confirmed using the Lilliefors test.

\subsection{Experiment 2}

\subsubsection{Participants}

Twelve right-handed stroke patients (two females, ten males) with an average age of $57.4 \pm 10.1$ years were recruited for this part of the study. None of the participants presented with any hearing deficiency. Inclusion criteria encompassed patients aged over 18 years having suffered from superior division MCA stroke in a period 3-24 months before the recruitment in the study; able to follow commands (no or limited cognitive impairment). Patients were excluded if they also presented with concomitant neurological or other severe medical problems, seizure history, cognitive impairments, treatment with drugs that act on central nervous system, complete paralysis of legs, cardiovascular or respiratory symptoms contraindicative of walking, contraindications to magnetic resonance imaging (MRI), cardiovascular or respiratory symptoms contraindicative of walking and any other significant non-stroke-related impairment affecting walking. All procedures were approved by the Ethics Committee of the Clinical Center of Serbia and all patients provided their written informed consent.

\subsubsection{Experimental set-up}

This was identical to Experiment 1, with the exception that only the control and the high attention condition were used, each with two repetitions. This choice was made since the results of Experiment 1 indicated that the high 
attention condition has a greater effect on MRCP parameters than the low attention condition (see more details in section 3). The experimental details are outlined in figure 2.

\subsubsection{ERP and MRCP analysis and MRCP feature extraction}

This was identical to the analysis and feature extraction as for Experiment 1.

\subsubsection{Statistics}

To determine the effect of the attention task on the MRCP, two-way analysis of variance (ANOVA) was used where 'Set' (first and second) and 'Attention status' (control and drifted attention) were used as fixed factors. The results were assumed significant if $p \leq 0.05$. The normality of the data was confirmed using the Lilliefors test. 


\section{Results}

Extracted features from single-trial MRCPs of $\mathrm{Cz}$ as well as from the large Laplacian filter were used to quantify the effect of attention on BCI performance. Since in the control condition, participants simply repeated the 90 dorsiflexion movements in three sets of 30 trials, we also quantified the effect of task repetition during the control and during the attention conditions.

\subsection{Experiment 1}

\subsubsection{ERP parameters}

Figure 3 depicts the P300 amplitude for the control, low and high attention conditions across all participants. The amplitude of the $\mathrm{P} 300$ for simple oddball $(5.9 \pm 2.8 \mu \mathrm{V})$ was significantly higher than for complex oddball $(4.4 \pm$ $\left.2.6 \mu \mathrm{V} ; \mathrm{F}_{(1,28)}=6.1, \mathrm{p}=0.02\right)$. Bonferroni post -hoc test revealed that the amplitude of the P300 was not significantly different between first and second set of each auditory oddball.

\subsubsection{MRCP features}

The MRCP parameters that were compared between the control and the two attention conditions were VPN, TPN, pre and post phased slopes and pre and post phase variability as outlined in section 2.6.

\subsubsection{Peak negativity comparison}

Two-way ANOVA revealed that VPN changed significantly between the control and high attention level $\left(\mathrm{F}_{(1,42)}=\right.$ 4.7, $\mathrm{p}=0.03)$ and among sets $\left(\mathrm{F}_{(2,42)}=3.9, \mathrm{p}=0.03\right)$. These results are presented in table 1. Post-hoc tests represented significant difference between the first and second set in the group with complex oddball task.

The grand average of the MRCP from each group (figure 4), visually illustrates that with task repetition in the control condition VPN and TPN did not change significantly. However, in the group with complex oddball, the VPN was decreased significantly after the first set of the oddball task. Statistical analysis revealed that the VPN for the control condition did not change significantly between set one, two and three of the movement task (simple oddball: $26.2 \pm 5.3 \mu \mathrm{V}, 24.5 \pm 5.2 \mu \mathrm{V}, 27.4 \pm 5.7 \mu \mathrm{V}$; complex oddball: $28.1 \pm 5.6 \mu \mathrm{V}, 27.6 \pm 06.5 \mu \mathrm{V}$, $28.4 \pm 6.6 \mu \mathrm{V})$. However, the peak negativity decreased significantly only in the high attention condition $\left(\mathrm{F}_{(2,21)}=\right.$ $3.5, p=0.048 ; 27.5 \pm 5.8,18.7 \pm 6.4,27.3 \pm 5.1 \mu \mathrm{V}$ with respect to set). Similarly, the imposed attention shifts did not have a significant effect on TPN for either the low or high attention condition $(\mathrm{p}>0.05)$.

\subsubsection{Pre and post phase slopes}


There was no significant effect on the pre or post phase slopes based on attention status and movement sets $(p>0.05)$.

\subsubsection{Pre-movement phase variability}

Pre-movement variability was extracted to explore the effect of attention on movement preparation. There was a significant increase in the variability from two to one seconds prior to TPN based on movement sets in the group performing the complex oddball task $\left(\mathrm{F}_{(2,42)}=4.3, \mathrm{p}=0.02\right)$. Post-hoc test revealed that the first and second set differed significantly $(\mathrm{p}=0.01)$. Figure 5 illustrates the variability in both groups for the entire data range extracted. The variability was significantly increased in the range of two to one seconds prior to TPN and only for the high attention condition $\left(\mathrm{F}_{(2,21)}=3.5, \mathrm{p}=0.048\right)$.

\subsubsection{Movement detection factors}

Two-way nested ANOVA revealed no significant interaction between the attention condition and task repetition for the three response factors, DL, TPR and FPR. Further, none of these variables were significantly different between the two attention levels in both groups of task complexity. However, DL and TPR were significantly different among sets (DL: $\mathrm{F}_{(2,42)}=4.5, \mathrm{p}=0.02$; TPR: $\left.\mathrm{F}_{(2,42)}=6.7, \mathrm{p}=0.003\right)$.

DL in the control condition did not change significantly within sets in both groups $(\mathrm{p}>0.05)$. However, this parameter was significantly different based on the movement set for the high attention condition $\left(\mathrm{F}_{(2,21)}=4.1\right.$, $\mathrm{p}=0.03$ ). Post-hoc analysis revealed a significant difference between the first and second set of the movement task in the high attention condition $(\mathrm{p}=0.04)$. Table 2 represents response factor values for the two groups of participants.

Although TPR values did not reveal any significant differences according to the set factor in the control condition for both groups, when the attention was shifted, TPR values changed significantly for the high attention condition $\left(\mathrm{F}_{(2,21)}=5.3, \mathrm{p}=0.01 ; 77.1 \pm 3.9,67.1 \pm 5.1,71.1 \pm 7.1\right.$ in relation to the three movement sets $)$. As for detection latency, the post-hoc test showed that there was a significant difference between the first and second set of the movement task but only in the high attention condition $(\mathrm{p}=0.01)$.

FPR measurements did not show a significant difference based on set in the control condition ( $p>0.05)$. However, FPR was increased significantly in the high attention condition $\left(\mathrm{F}_{(2,21)}=5.2, \mathrm{p}=0.01\right)$ after the first set of the auditory oddball task.

\subsubsection{Laplacian results}

Aside from detection parameters being calculated for the single channel $\mathrm{Cz}$, we also applied the large Laplacian. Two-way ANOVA revealed no significant difference in MRCP features or detection parameters for either the 
attention levels or movement sets ( $>>0.05)$. None of the MRCP features that were VPN, TPN, variability and slopes were changed between attention levels in both oddball experiments. These results are presented in table 2 and 3.

\subsection{Experiment 2}

\subsubsection{Peak negativity comparison}

Figure 6 (a) and (b), shows the grand average of the MRCP across all patients for both the control and the high attention condition. Two-way ANOVA revealed a significant decrement of VPN between the control and the high attention condition $\left(\mathrm{F}_{(1,40)}=5.1, \mathrm{p}=0.03\right)$ as well as between sets of the movement task $\left(\mathrm{F}_{(1,40)}=4.9, \mathrm{p}=\right.$ 0.03). Statistical analysis on negative peak time indicated that attention did not have a significant effect on TPN in the patients.

\subsubsection{Pre and post phase slopes}

Based on the results of the two-way ANOVA, the slope values in the range of two seconds prior to peak negativity up to peak negativity decreased significantly both between conditions $\left(\mathrm{F}_{(1,40)}=5.5, \mathrm{p}=0.02 ;-11.6 \pm 4.8\right.$ and $-8.7 \pm 3.3$ for the control and attention conditions respectively) and also among movement sets $\left(\mathrm{F}_{(1,40)}=5, \mathrm{p}=\right.$ $0.03 ;-11.6 \pm 4.5$ in first set and $-8.7 \pm 3.9$ in the second set of the movement task) (figure $6 \mathrm{a}$ and $6 \mathrm{~b}$ ). One-way ANOVA revealed that the effect between movement sets was significant only in the attention condition $\left(\mathrm{F}_{(1,20)}=\right.$ $6.2, p=0.02 ;-10.5 \pm 3.7$ and $-7.7 \pm 3.2$ for the first and second set of the movement task respectively). In addition, slope values in the range of one second prior to the point of peak negativity were significantly different when the attention was drifted $\left(\mathrm{F}_{(1,40)}=6.2, \mathrm{p}=0.02\right)$ and also based on movement sets $\left(\mathrm{F}_{(1,40)}=5, \mathrm{p}=0.03\right)$. The repetition of the movement task had a significant effect on this slope parameter only in the high attention condition $\left(\mathrm{F}_{(1,20)}\right.$ $=5.5, \mathrm{p}=0.03)$. The slope within the rebound rate phase was not significantly different between conditions $\left(\mathrm{F}_{(1,40)}<1, \mathrm{p}>0.05\right)$.

\subsubsection{Pre-phase variability}

As for experiment 1, pre-movement variability was extracted to explore the effect of attention on movement preparation. Figure $6 \mathrm{c}$ and $6 \mathrm{~d}$ illustrates the variability for both the control and high attention condition for the entire data range extracted while table 1 reports the values of the variability within the different time windows investigated.

The variability in the range of two to one seconds prior to TPN was significantly different based on attention level $\left(\mathrm{F}_{(1,40)}=14, \mathrm{p}=0.001\right)$ and also among movement sets $\left(\mathrm{F}_{(1,40)}=6.6, \mathrm{p}=0.01\right)$. The variability was 
significantly increased in the range of two to one seconds prior to TPN for the high attention condition $\left(\mathrm{F}_{(1,20)}\right.$ $=7.6, \mathrm{p}=0.01)$.

\subsubsection{Movement detection factors}

DL was changed significantly when the attention was drifted $\left(\mathrm{F}_{(1,40)}=7.1, \mathrm{p}=0.01\right)$ and based on movement sets $\left(\mathrm{F}_{(1,40)}=12.6, \mathrm{p}=0.001\right)$. According to the results of the one-way ANOVA no significant changes were found in the control condition between the first and second movement sets $\left(\mathrm{F}_{(1,20)}=1.4, \mathrm{p}>0.05\right)$. However for the high attention task, task repetition had a significant effect on $\mathrm{DL}\left(\mathrm{F}_{(1,20)}=6.4, \mathrm{p}=0.03\right.$; see also table 2).

TPR values changed significantly only between the control and the high attention condition $\left(\mathrm{F}_{(1,40)}=4.9, \mathrm{p}=0.03\right)$ but not between movement sets. TPR decreased significantly $\left(\mathrm{F}_{(1,20)}=10.9, \mathrm{p}=0.004\right)$ for the high attention conditions compared to the control condition (table 2).

For FPR, only movement repetition had a significant effect $\left(\mathrm{F}_{(1,40)}=4.8, \mathrm{p}=0.03\right)$. One-way ANOVA revealed that FPR values significantly increased based on movement repetition only in the high attention condition $\left(\mathrm{F}_{(1,20)}\right.$ $=5.6, \mathrm{p}=0.03)$.

\subsubsection{Laplacian results}

As for Experiment 1, the large Laplacian with $\mathrm{Cz}$ as the central channel was used to analyze the data. Based on this method, none of the nine temporal features and detection parameters showed a significant effect of attention diversion. The results are shown in table 2 and 3. 


\section{Discussion}

In the current study we investigated the influence of imposed shifts in attention interlaced with three sets of a dorsiflexion task on specific characteristics of the MRCP. A decrement in the user attention resulted in a significant delay in the detection of movement intention but only when the single channel $(\mathrm{Cz})$ was used. This has important implications for BCIs designed for neuromodulation. Here the user's motor intent is detected online and used to trigger an external device that produces the intended movement. In the past, our group and others have shown that the timing between detection and the triggering of the external device is decisive for the induction of plasticity based on Hebbian theory of associativity (Mrachacz-Kersting et al., 2012; Xu et al., 2014). Equally important is the effect that low attention has on the induction of this type of plasticity. If attention is low then no plasticity is evoked, irrespective of the correct association between the intent and the reproduction of that movement (Stefan et al., 2004; Ziemann et al., 2008). Thus the results presented here highlight the importance of online single channel monitoring for drifts in attention that cannot be revealed by the more robust Laplacian approach. If the large Laplacian was implemented, movement detection parameters did not differ between control and the shifted attention conditions. For a robust BCI both in terms of optimal performance in movement detection but also in detection of attention shift, a hybrid BCI would likely be an optimal solution. That is, movement is detected using the large Laplacian and is complemented by detection of attention shifts using only a single channel. Thus in future designs of a BCI for neurorehabilitation we need to include, both of these signal modalities for real-time applications: High detection accuracy (which Laplacian can provide even if attention is altered), and attention to the main task must be monitored (which we have shown here can be done using the single channel $\mathrm{Cz}$ ).

\subsection{Attention and task repetition}

In this study, an auditory oddball paradigm with two levels of difficulty was used to experimentally alter the users' attention. In principle, both visual and auditory oddball paradigms will reveal differences between attended or unattended stimuli in various brain signals such as ERP and mismatch negativity (MMN) (Barkaszi et al., 2013; Wei et al., 2002; Wilson et al., 2012). However, the P300 amplitude evoked using visual oddball paradigms has a smaller amplitude compared to that induced by an auditory stimulus. In our initial pilot experiments we confirmed these results and chose the auditory oddball paradigm for the current study. In accordance with the previous studies (Wilson et al., 2012; Wintink et al., 2001), the P300 amplitude was larger when more attention was needed and decreased when the task was more complex (figure 3).

Dual tasking is modulating two tasks concurrently and is therefore more complex than performing single tasks. In these cases, one of the tasks which requires more attention is the primary (main) task and the other referred to 
as the secondary task functions as a distractor for the primary task (Wilson et al., 2012). In daily life, attention can be diverted due to many reasons such as performing two tasks simultaneously or switching between two different tasks. In the current study we could have combined the visual task with the auditory oddball task, thus dividing the attention between two tasks, however we separated these in our protocol to initially quantify the influence of switching attention between two tasks when one task is completed and attention is diverted to the other task. According to the MRCP results, attention to the dorsiflexion task was influenced by the oddball task since movement preparation and execution parameters were significantly affected.

Reduction in the attentional load can delay motor execution (Tard et al., 2014) since it has a significant effect on working memory and procedural learning (Ziemann et al., 2008). In our experiment, when participants were asked to complete the auditory oddball task between dorsiflexion sets, their attentional focus was decreased internally, thus likely leading to a diminished learning process (Johnson et al., 2013). One of the aims of this study was to explore the effect of task repetition on cortical signal characteristics (Brunet et al., 2014). Although the results indicate that movement repetition caused improved performance, it was shown to have no significant effect on either pre or post-movement features of the MRCP. Stimulus repetition leads to task learning and a reduction in neural activity and this has been associated to improved task performance (Grill-Spector et al., 2006). Since the frequency and timing of the auditory oddball were the same in the two sets, the amount of new information was reduced in the third compared to the second set, likely leading to the participants becoming habituated to the task (Ritter et al., 1968). This may have allowed them to maintain their focus within the third set of dorsiflexion. Also, task repetition is one of the possible reasons for decreases in memory load since it familiarizes participants to the movement through enhanced recognition memory (Brown and Xiang, 1998). The stimulus that has been seen many times previously can reduce neural response time as quantified in the current study with improved movement detection. Figure 4 presents the effect of repetition on task improvement also when the attention was drifted. This effect was the same for the patient group (figure 6) during the normal attention state. A possible reason for a non-significant effect of repetition may be attributed to task complexity. If a task is complex, it requires more repetitions until the task is learned (Jordan and Rabbitt, 1977; Perez et al., 2004).

Attention plays a key role in processing resources and its effect on attention is not only restricted to the stimulus processing but also includes attention to the motor processes and trains of thoughts (Purves et al., 2008). In the current study, attention was altered with the single oddball task while performing a simple motor task as a main task. These tasks were separated into different experimental runs to avoid the effect of dual-tasking and to understand the influence of interference of a secondary (oddball) task in attention diversion. Results show that the level of the attention task (simple vs complex) significantly influences performance of the main task 
performance. Thus when participants were required to focus more on the attention task, detection performance for the main task was reduced. Likely this effect will be further enhanced when the two tasks are interlaced.

Aside from the task level difficulty, mental or cognitive fatigue may reduce attention focus to the main task and thus task performance (Faber et al., 2012; Van der Linden, 2011). There are several reasons why we believe that participants did not experience fatigue. Firstly, this type of fatigue is typically reported only for long-time monotonous tasks (Wascher et al., 2016) and in the case of our paradigm there were at least two tasks performed interchangeably. Second, there were not significant differences among repetitions of the control level, thus for at least this group, fatigue was not an issue. Thirdly, the study was designed such that the attention conditions (control and diverted attention) were counterbalanced so as to exclude that any observed significant differences were due to fatigue. For the patient group, the experiment was shortened by only investigating a control and a high attention level.

\subsection{BCI for neuromodulation}

Neuromodulation feedback in BCI systems and brain plasticity are depended on movement detection latency and accuracy but also influenced by attention of the BCI user (Ziemann et al., 2008). In the present study, the MRCP from a single channel as well as a combination of nine channels when using Laplacian were used to quantify the effect of attention alternation. Statistical analysis revealed a significant difference of the peak negativity value when the attention was shifted in both healthy and patient participants only in single channel MRCPs. This suggests that movement preparation and decision making for task execution, which depends on cognitive processing, are decreased with a reduction of attention. Several studies (McCallum and Walter, 1968; Tard et al., 2014; Tecce et al., 1976) have suggested that the relation between attention and MRCP magnitude is positive and monotonic. Since in the current study the MRCP amplitude was reduced with an increasing shift in attention, it may serve as a future marker for attentional shifts. The functionality and connectivity within the neural networks is delayed when the attentional level is reduced, so, we can also expect increases in detection of movement intent and reaction time (Lu et al., 2012). Indeed this is what we found and it is likely associated to the greater MRCP variability when attention is drifted (Figure 5). As demonstrated in previous studies, signal variability is an indicator of movement preparation processes (Aliakbaryhosseinabadi et al., 2014; Jiang et al., 2015 ) and it is likely that these are most affected by the alterations in user attention.

On average, 5 trials out of a total of 30 were removed due to artifact contamination such as eye blinks and head movements. In a real-time BCI this would lead to a decrease in the number of detected movement intents and thus neurofeedback to the user. In our previous studies we have shown that plasticity is still induced with a similar number of rejected trials (Xu et al., 2014) . 
Surprisingly, the MRCP variations were significant only when extracted from a single sensorimotor channel $(\mathrm{Cz})$ but not when using a spatial filtering method such as large Laplacian filter. One of the possible reasons may be linked to the type of experiment, which focused on the attention effect on motor task execution. In previous studies (Gómez et al., 2006; Krusienski et al., 2012) it has been shown that it is difficult to define an appropriate channel combination for the extraction of effective parameters on EEG signals. For instance, a preparatory attention effect has been reported to affect the frontal and occipital lobe only (Purves, 2008). The application of the large Laplacian filter thus smoothed out the signal variations since it uses the surrounding channels of the main channel.

\section{Conclusion}

In this study we aimed to explore the effect of internal attention diversion on movement execution characteristics in both healthy and stroke participants. Results show that attention diversion can delay movement intent detection in both healthy participants and stroke patients, although it has a greater effect on the movement preparation phase in the patient group. Furthermore, it was revealed that habituation of movement performance can be influenced by attention changes. Results of this study were obtained based on offline data analysis but the next step would be online detection. We are currently working towards combining the two tasks to investigate the effect of dual-tasking on attention diversion. 


\section{References}

Albares M, Criaud M, Wardak C, Nguyen SCT, Ben Hamed S, Boulinguez P. Attention to baseline: Does orienting visuospatial attention really facilitate target detection. J Neurophysiol 2011; 106:809-16.

Aliakbaryhosseinabadi S, Jiang N, Vuckovic A, Lontis R, Dremstrup K, Farina D, et al.. Detection of movement intention from movement-related cortical potentials with different paradigms. Proc 2nd Int Conf NeuroRehabilit (ICNR) 2014; 7: 237-44.

Barkaszi I, Czigler I, Balázs L. Stimulus complexity effects on the event-related potentials to taskirrelevant stimuli. Biol Psychol 2013; 94: 82-9.

Bentin S, McCarthy G. The effects of immediate stimulus repetition on reaction time and event-related potentials in tasks of different complexity. J Exp Psycho Learn Mem Cogn 1994; 20: 130-49.

Brown MW, Xiang J. Recognition memory: Neuronal substrates of the judgement of prior occurrence. Prog Neurobiol 1998; 55:149-189.

Brunet NM, Bosman CA, Vinck M, Roberts M, Oostenveld R, Desimone R, et al.. Stimulus repetition modulates gamma-band synchronization in primate visual cortex. Proc Natl Acad Sci USA 2014; 111: 3626-31.

Desimone, R. Neural mechanisms for visual memory and their role in attention. Proc Nat Acad Sci USA 1996; 93: 13494-99.

Esghaei M, Daliri MR. Decoding of visual attention from LFP signals of macaque MT. PLoS ONE, 2014; 9: e100381

Faber LG, Maurits NM, Lorist MM. Mental fatigue affects visual selective attention. PLoS ONE, 2012; 7 : e48073

Gómez, CM, Marco-Pallarés J, Grau C. Location of brain rhythms and their modulation by preparatory attention estimated by current density. Brain Res 2006; 1107: 151-60.

Grill-Spector K, Henson R, Martin A. Repetition and the brain: Neural models of stimulus-specific effects. Trends Cogn Sci 2006; 10: 14-23.

Gu Y, Farina D, Murguialday AR, Dremstrup K, Birbaumer N. Comparison of movement related cortical potential in healthy people and amyotrophic lateral sclerosis patients. Front Neurosci 2013; 7: 65 .

Hallett M. Movement-related cortical potentials. Electromyogr Clin Neurophysiol 1994; 34: 5-13. 
Jiang N, Gizzi L, Mrachacz-Kersting N, Dremstrup K, Farina D. A brain-computer interface for singletrial detection of gait initiation from movement related cortical potentials. Clinic Neurophysiol 2015; 126:154-9.

Johnson L, Burridge JH, Demain SH. Internal and external focus of attention during gait re-education: An observational study of physical therapist practice in stroke rehabilitation. Phys Ther 2013; 93: 957-66.

Jordan TC, Rabbitt PM. Response times to stimuli of increasing complexity as a function of ageing. Br J Psychol 1977; 68: 189-201.

Kimura M, Katayama J, Murohashi H. Effects of feature and spatial attention on visual change detection. Neuroreport 2008; 19: 389-92.

Koyas E, Hocaoglu E, Patoglu V, Cetin M. Detection of intention level in response to task difficulty from EEG signals. Machine Learning for Signal Processing (MLSP) IEEE International Workshop 2013; 2013: 1-6.

Krusienski DJ, McFarland DJ, Wolpaw JR. Value of amplitude, phase, and coherence features for a sensorimotor rhythm-based brain-computer interface. Brain Res Bull, 2012; 87:130-4.

Lu M, Arai N, Tsai C, Ziemann U. Movement related cortical potentials of cued versus self-initiated movements: Double dissociated modulation by dorsal premotor cortex versus supplementary motor area rTMS. Hum Brain Mapp 2012; 33: 824-39.

McCallum WC, Walter G. The effects of attention and distraction on the contingent negative variation in normal and neurotic subjects. Electroencephalogr Clin Neurophysiol 1968; 25: 319-29.

McFarland DJ, McCane LM, David SV, Wolpaw JR. Spatial filter selection for EEG-based communication. Electroencephalogr Clinic Neurophysiol 1997; 103: 386-94.

Mrachacz-Kersting N, Jiang N, Stevenson AJT, Niazi IK, Kostic V, Pavlovic A, et al.. Efficient neuroplasticity induction in chronic stroke patients by an associative brain-computer interface. $\mathrm{J}$ Neurophysiol 2015; 115:1410-21.

Mrachacz-Kersting N, Kristensen SR, Niazi IK, Farina D. Precise temporal association between cortical potentials evoked by motor imagination and afference induces cortical plasticity. J Physiol 2012; 590: 1669-82.

Pedroso RV, Fraga FJ, Corazza DI, Andreatto CA, Coelho FG, Costa JL, et al.. P300 latency and amplitude in alzheimer's disease: A systematic review. Braz J Otorhinolaryngol 2012; 78 : 126-32.

Perez MA, Lungholt BKS, Nyborg K, Nielsen JB. Motor skill training induces changes in the excitability of the leg cortical area in healthy humans. Exp Brain Res 2004; 159: 197-205. 
Purves D. Principles of cognitive neuroscience. 2nd ed. Sinauer Associates, Inc.; 2008.

Ritter W, Vaughan HG, Costa LD. Orienting and habituation to auditory stimuli: astudy of short term changes in average evoked responses. Electroencephalogr Clin Neurophysiol 1968; 25: 550-6

Roy RN, Bonnet S, Charbonnier S, Campagne A. Mental fatigue and working memory load estimation: Interaction and implications for EEG-based passive BCI Conf Proc IEEE Eng Med Biol Soc 2013; 2013: 6607-10

Scheel H, Xu R, Jiang N, Mrachacz-Kersting N, Dremstrup K, Farina D. Influence of external cues on synchronized brain-computer interface based on movement related cortical potentials. NER Conf IEEE EMBS 2015; 7:45-8.

Shenoy P, Krauledat M, Blankertz B, Rao RPN, Müller K. Towards adaptive classification for BCI. J Neural Eng 2006; 3: 13-23.

Stefan K, Wycislo M, Classen J. Modulation of associative human motor cortical plasticity by attention. J Neurophysiol 2004; 92: 66-72.

Tard C, Dujardin K, Bourriez J, Destée A, Derambure P, Defebvre L, et al.. Attention modulates step initiation postural adjustments in parkinson freezers. Parkinsonism Relat Disord 2014; 20: 284-89

Tecce JJ, Savignano-Bowman J, Meinbresse D. Contingent negative variation and the distractionarousal hypothesis. Electroencephalogr Clin Neurophysiol 1976; 41: 277-86.

Toppi J, Risetti M, Quitadamo LR, Petti M, Bianchi L, Salinari S, et al.. Investigating the effects of a sensorimotor rhythm-based BCI training on the cortical activity elicited by mental imagery. J Neural Eng 2014; 11: 035010.

Treder MS, Purwins H, Miklody D, Sturm I, Blankertz B. Decoding auditory attention to instruments in polyphonic music using single-trial EEG classification. J Neural Eng 2014; 11: 026009.

Van der Linden D. The urge to stop: The cognitive and biological nature of acute mental fatigue. In P. L. Ackerman (Ed.), Cognitive fatigue: Multidisciplinary perspectives on current research and future applications, (pp. 149-164). Washington, DC, US: American Psychological Association

Xu R, Jiang N, Lin C, Mrachacz-Kersting N, Dremstrup K, Farina D. Enhanced low-latency detection of motor intention from EEG for closed-loop brain-computer interface applications. IEEE Trans Biomed Eng 2014; 61: 288-96.

Xu R, Jiang N, Mrachacz-Kersting N, Dremstrup K, Farina D. Factors of influence on the performance of a short-latency non-invasive brain switch: evidence in healthy individuals and implication for motor function rehabilitation. Front Neurosci 2016; 9:9. 
Wang R, Dong Z, Chen X, Zhang M, Yang F, Zhang X, et al.. Gender differences of cognitive function in migraine patients: evidence from event-related potentials using the oddball paradigm. J Headache Pain 2014; 15: 6.

Wascher E, Getzmann S, Karthaus M. Driver state examination-Treading new paths. Accid Anal Prev, 2016; 91: 157-165

Wei JH, Chan TC, Luo YJ. A modified oddball paradigm “cross-modal delayed response" and the research on mismatch negativity. Brain Res Bull 2002; 57: 221-30.

Wilson MJ, Harkrider AW, King KA. The Effects of Visual Distracter Complexity on Auditory Evoked P3b in Contact Sports Athletes. Dev Neuropsuchol 2014; 39: 113-30.

Wintink AJ, Segalowitz SJ, Cudmore LJ. Task complexity and habituation effects on frontal P300 topography. Brain Cong 2001; 46: 307- 11

Zander TO, Jatvez S. Context-aware brain-computer interfaces: exploring the information space of user, technical system and environment. J Neural Eng 2012; 9: 016003.

Ziemann U, Paulus W, Nitsche MA, Pascual-Leone A, Byblow WD, Berardelli A, et al.. Consensus: Motor cortex plasticity protocols. Brain Stimul 2008; 3: 164-82. 
Figure 1. (a)Experiment set up for recording movement-related cortical potentials with normal attention state without hearing auditory oddball (LEFT) and drifted attention state with interspersing auditory oddball (RIGHT).(b) Experimental paradigm during movement recording which consisted of five time phases. Participants had to start their movement when triangle reached to the ramp. (c) Illustration of nine features were extracted from each trial of EEG signals. 20 represents time domain of 2 seconds before TPN to place of TPN, 10 shows time range of 1 second prior to TPN to this point and 21 indicates 2 to 1 second before TPN.

Figure2. Illustration of experiment 1and experiment 2. Experiment1 consisted of two main levels that are control and attention level for two groups which are different in level of attention. In the control level of both groups participants had to do 3 blocks of cue based movement. Each block consisted 30 trials of cue-based motor execution which was separated from the other blocks with 4 min break time. In the attention level for both groups, each block of 30 trials movement execution was separated from the others by an auditory oddball paradigm. Experiment 2 consisted of one groups of patients with two levels of attention which are control and drifted attention level. In this experiment participants had two sets of task movement in each attention level that were separated with break or oddball paradigm.

Figure 3. Event related brain potentials elicited by target stimuli in both levels of attention drift. P3 amplitude is higher in simple oddball as comparing with the complex oddball. P3 amplitude is $4.7 \pm 3.1 \mu \mathrm{V}$ and $4 \pm 2.6 \mu \mathrm{V}$ for first and second repetition in complex oddball and $6.8 \pm 2.6 \mu \mathrm{V}$ and $6.1 \pm 2.1 \mu \mathrm{V}$ for first and second repetition in simple oddball. However P3 latency did not differ significantly among these repetitions.

Figure 4. Grand average of MRCP with two levels of attention in two drift attention groups. (a) and (b) are corresponded to control and attention level of low attention drift group and (c) and (d) are illustration of control and drifted attention of high attention drift. TPN was occurred nearly at $-100 \mathrm{~ms}$ for both (a) control and (b) attention level and the VPN was nearly the same in both control level but in (d) high attention group it is increased from $-27.5 \pm 5.8 \mu \mathrm{V}$ in first repetition to $-18.7 \pm 6.4 \mu \mathrm{V}$ in the second one.

Figure 5. Sample of variation among trials of one subject before and after drift attention within two groups of attention drift. (a) illustrates changes of attention in low attention drift group and (b) represents changes of variability in high attention drift group. The variability was changed in high attention drift in domain of [-2 -1] while this variability seems not to be significant in low attention drift. Average of Variability of high attention group in this time domain was $17,22,19 \mu \mathrm{V}$ for first, second and the third repetition of movement. But it is 13 , $14,10 \mu \mathrm{V}$ for first, second and the third repetition of low attention.

Figure 6. (a) and (b) represent grand average of MRCP of stroke patient with two levels of attention. (a) Two repetitions of movement in normal attention level while VPN is around $-19.8 \pm 12.2 \mu \mathrm{V}$ occurred nearly at $78 \mathrm{~ms}$ after movement onset. (b) Two repetitions of diverted attention level when VPN is about $-9.8 \pm 7.7 \mu \mathrm{V}$ and occurred at $103 \mathrm{~ms}$ after movement onset. (c) and (d) shows sample of variability in two repetitions within patient group in both levels of attention. (c) the variability in control level and (d) the variabity in attention level. The variability was increased in domain of [-2 -1] before TPN in attention level. TPN is the place of minimum variability. 
Table 1. Mean values and the corresponding $\mathrm{p}$ values of the single feature comparison in channel $\mathrm{Cz}$ between the two attention levels in the complex oddball tasks.

Table 2. Response factor of movement detection in drifted attention situations for healthy and patients and also for two levels of task demand from single channel $\mathrm{Cz}$ and its surrounding channel resulted of using large Laplacian.

Table 3. Mean values and the corresponding $\mathrm{p}$ values of the single feature comparison in Laplacian of $\mathrm{Cz}$ between the two attention levels in the complex oddball tasks. 


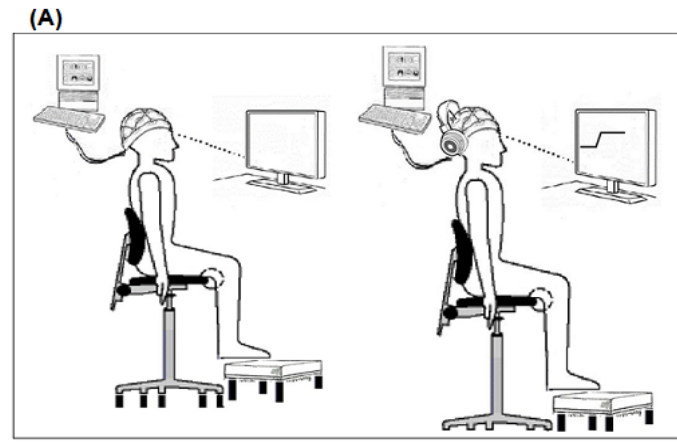

(B)

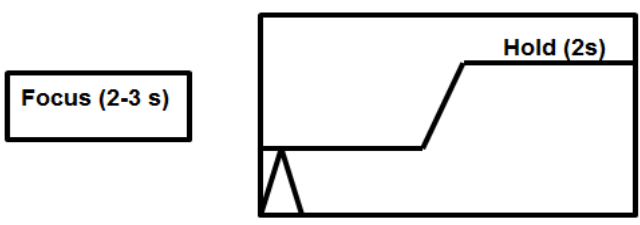

Rest (3-5 s)

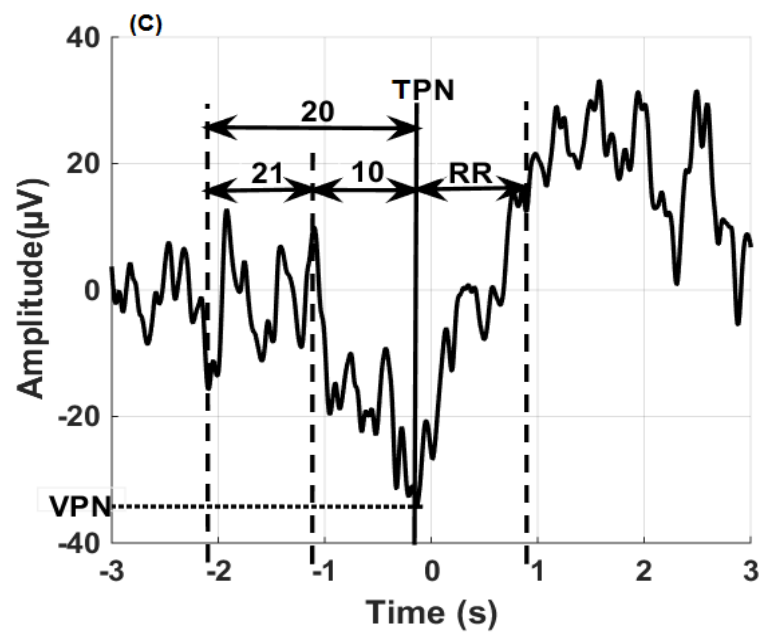

Figure 1. (a)Experiment set up for recording movement-related cortical potentials with normal attention state without hearing auditory oddball (LEFT) and drifted attention state with interspersing auditory oddball (RIGHT).(b) Experimental paradigm during movement recording which consisted of five time phases. Participants had to start their movement when triangle reached to the ramp. (c) Illustration of nine features were extracted from each trial of EEG signals. 20 represents time domain of 2 seconds before TPN to place of TPN, 10 shows time range of 1 second prior to TPN to this point and 21 indicates 2 to 1 second before TPN. 


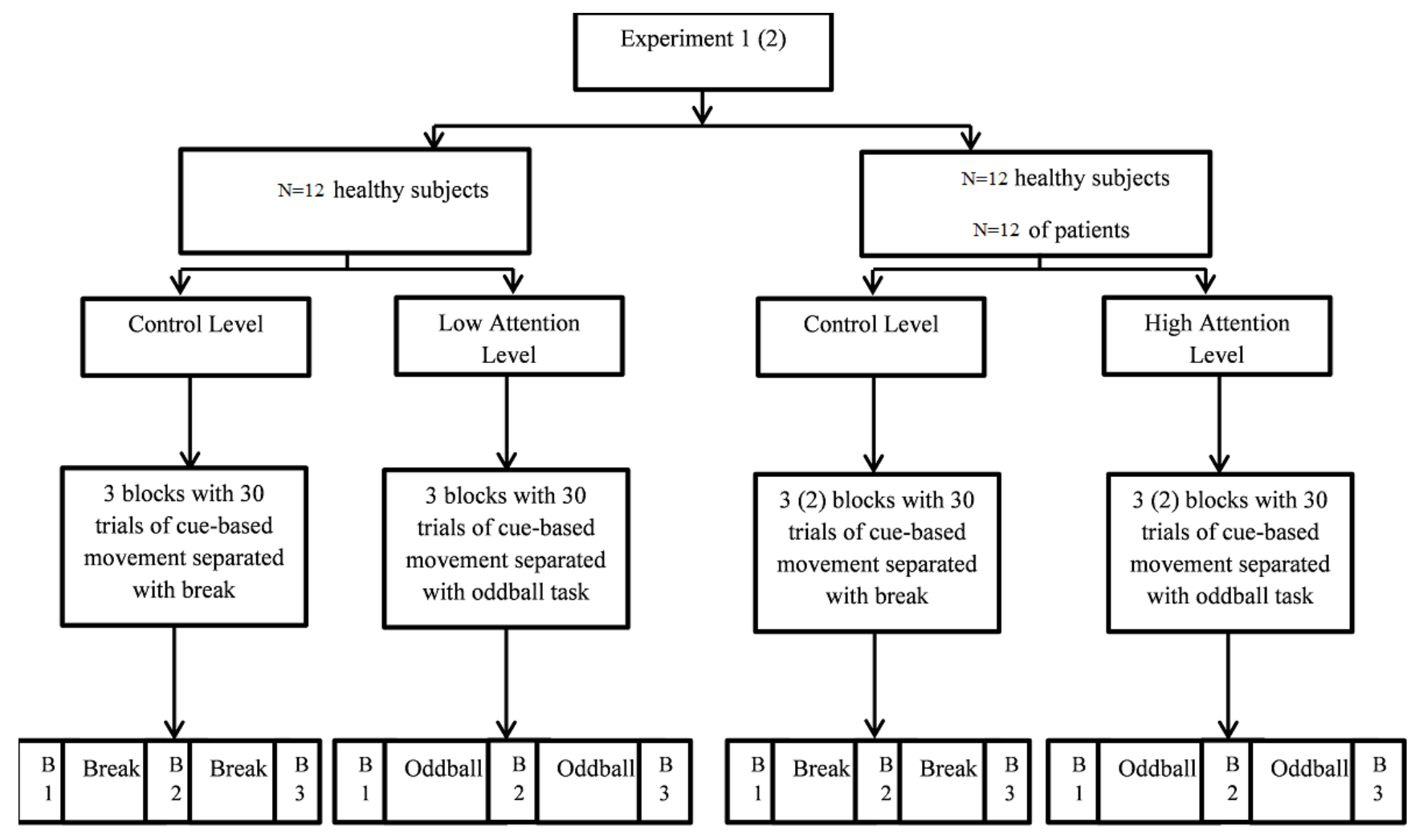

Figure2. Illustration of experiment1and experiment 2. Experiment 1 consisted of two main levels that are control and attention level for two groups which are different in level of attention. In the control level of both groups participants had to do 3 blocks of cue based movement. Each block consisted 30 trials of cue-based motor execution which was separated from the other blocks with 4 min break time. In the attention level for both groups, each block of 30 trials movement execution was separated from the others by an auditory oddball paradigm. Experiment 2 consisted of one groups of patients with two levels of attention which are control and drifted attention level. In this experiment participants had two sets of task movement in each attention level that were separated with break or oddball paradigm. 


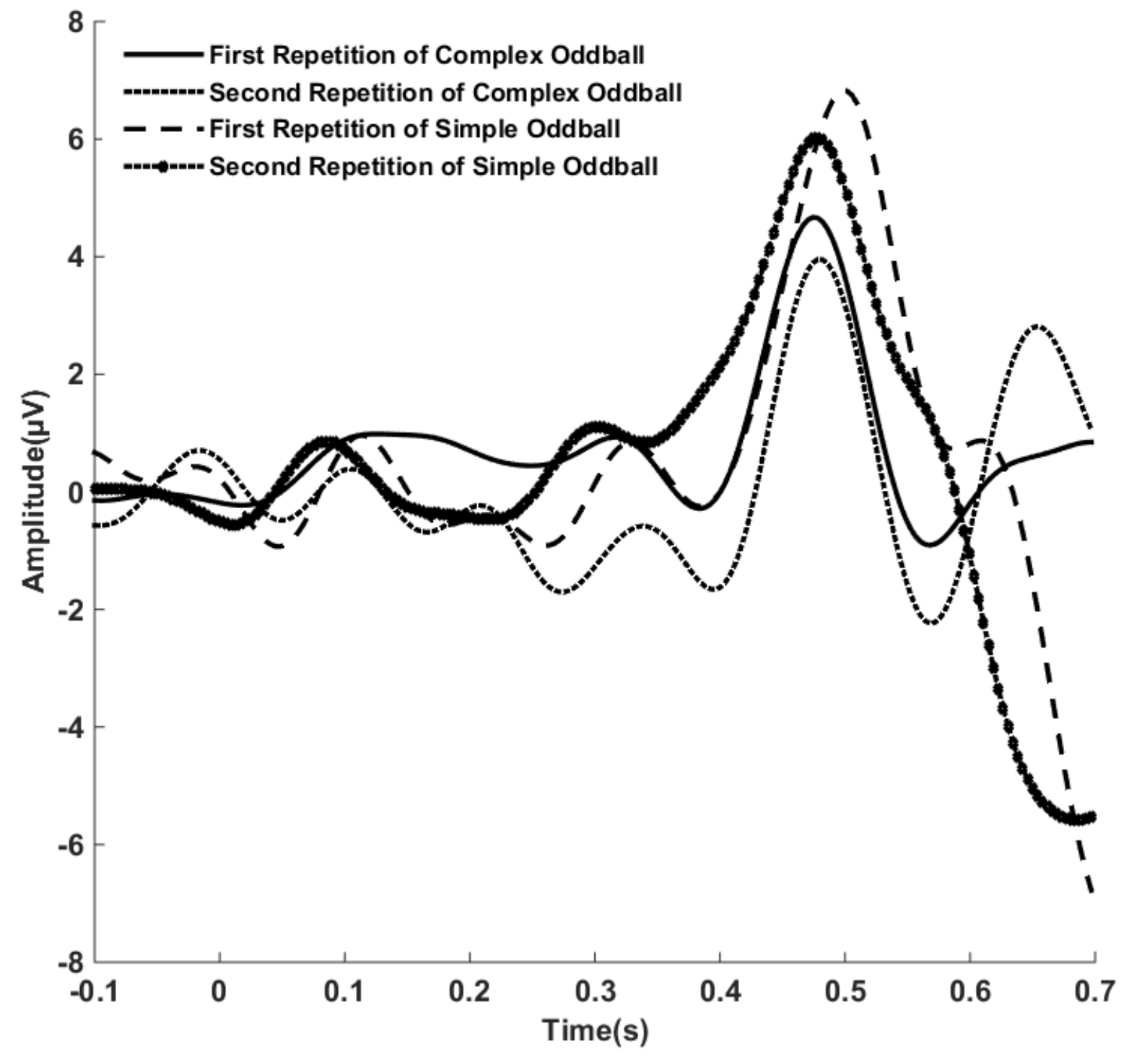

Figure 3. Event related brain potentials elicited by target stimuli in both levels of attention drift. P3 amplitude is higher in simple oddball as comparing with the complex oddball. P3 amplitude is $4.7 \pm 3.1 \mu \mathrm{V}$ and $4 \pm 2.6 \mu \mathrm{V}$ for first and second repetition in complex oddball and $6.8 \pm 2.6 \mu \mathrm{V}$ and $6.1 \pm 2.1 \mu \mathrm{V}$ for first and second repetition in simple oddball. However P3 latency did not differ significantly among these repetitions. 

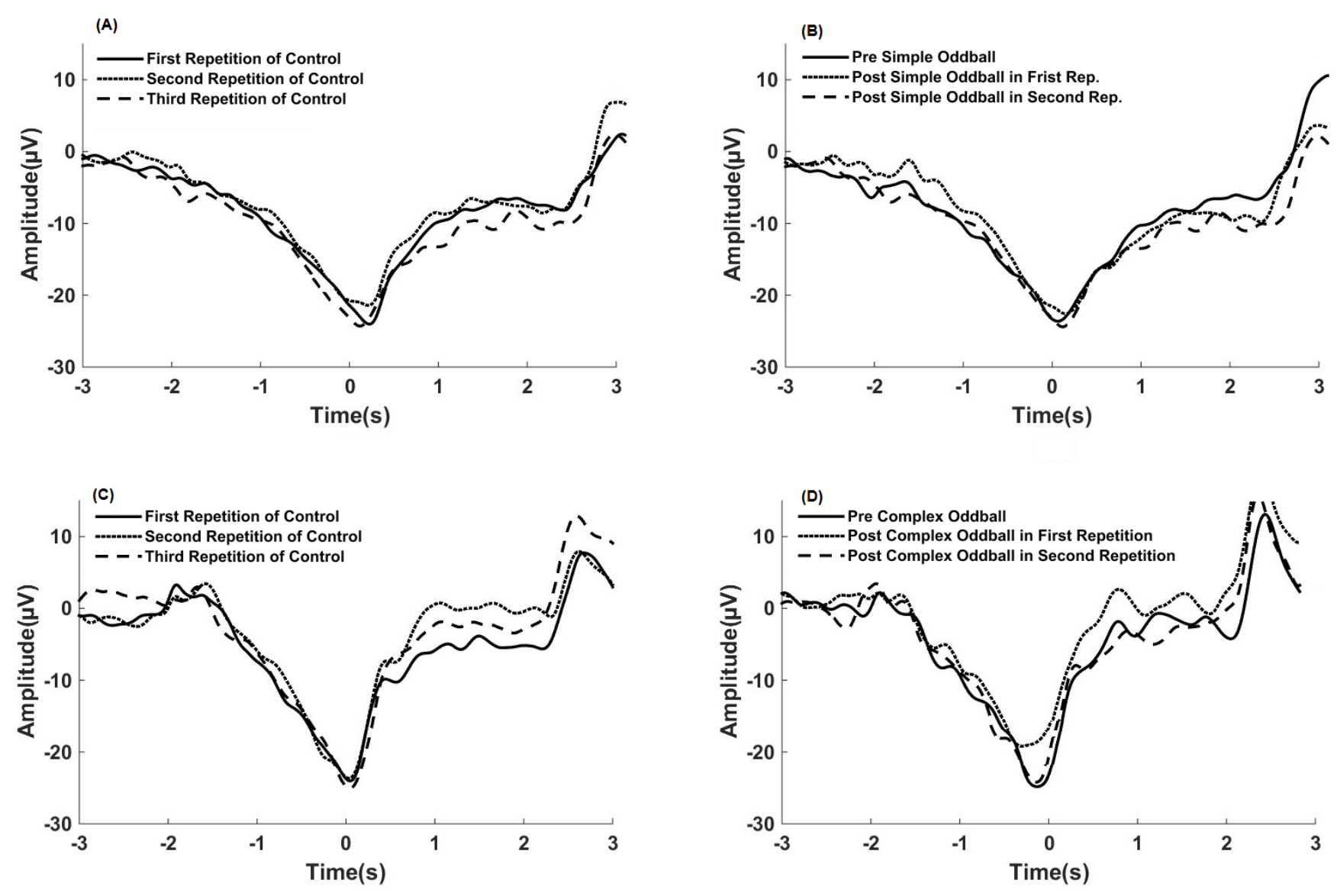

Figure 4. Grand average of MRCP with two levels of attention in two drift attention groups. (a) and (b) are corresponded to control and attention level of low attention drift group and (c) and (d) are illustration of control and drifted attention of high attention drift. TPN was occurred nearly at $-100 \mathrm{~ms}$ for both (a) control and (b) attention level and the VPN was nearly the same in both control level but in (d) high attention group it is increased from $-27.5 \pm 5.8 \mu \mathrm{V}$ in first repetition to $-18.7 \pm 6.4 \mu \mathrm{V}$ in the second one. 

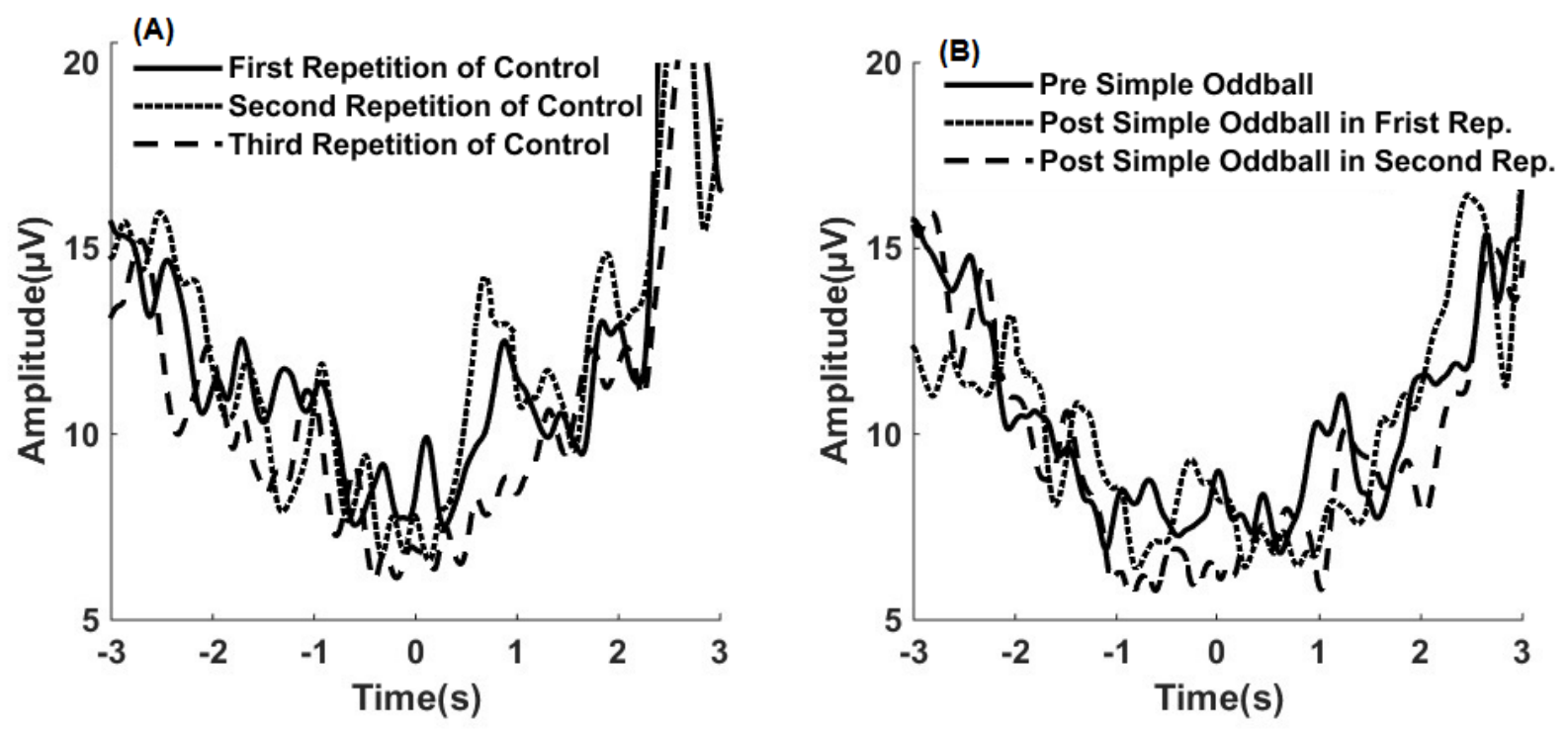

(C)
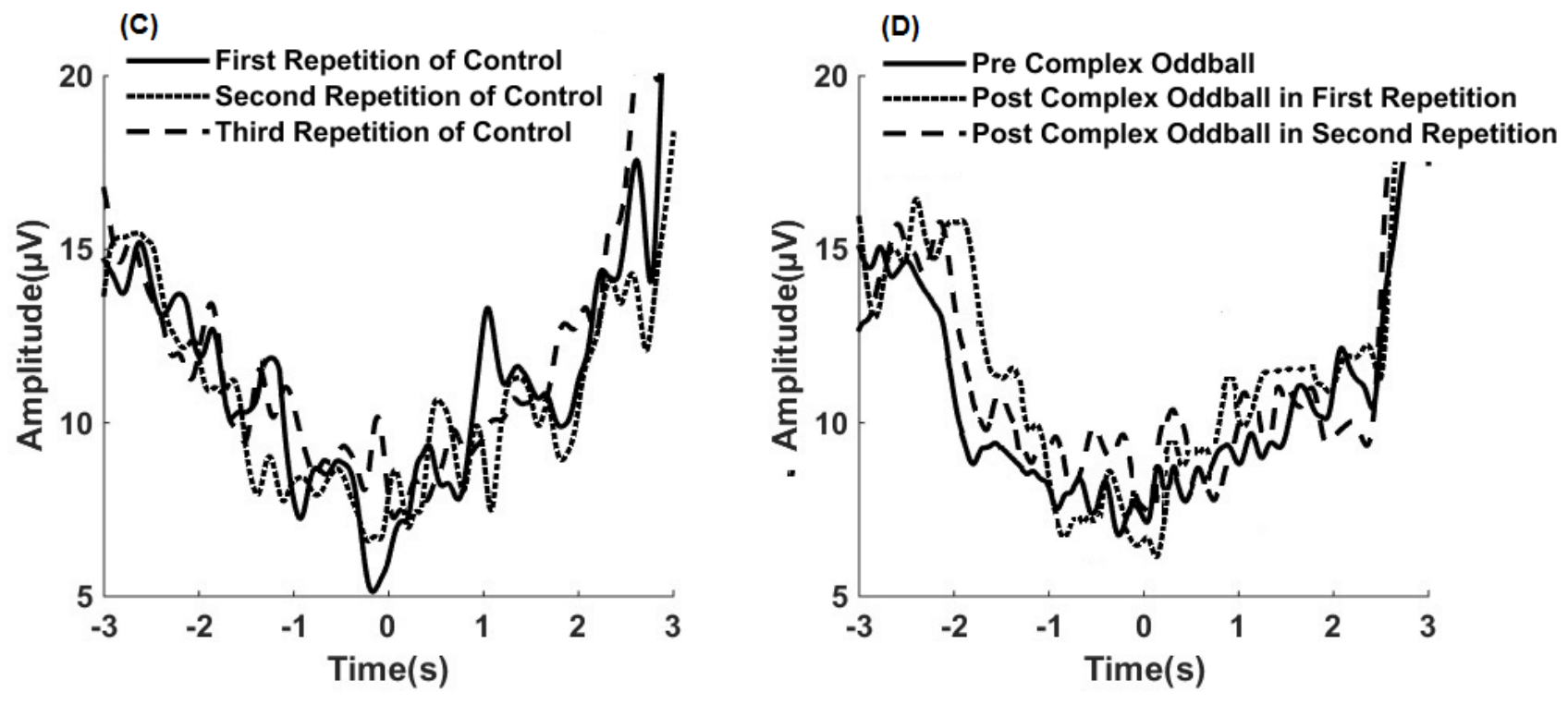

Figure 5. Sample of variation among trials of one subject before and after drift attention within two groups of attention drift. (a) illustrates changes of attention in low attention drift group and (b) represents changes of variability in high attention drift group. The variability was changed in high attention drift in domain of [-2 -1] while this variability seems not to be significant in low attention drift. Average of Variability of high attention group in this time domain was $17,22,19 \mu \mathrm{V}$ for first, second and the third repetition of movement. But it is 13 , $14,10 \mu \mathrm{V}$ for first, second and the third repetition of low attention. 

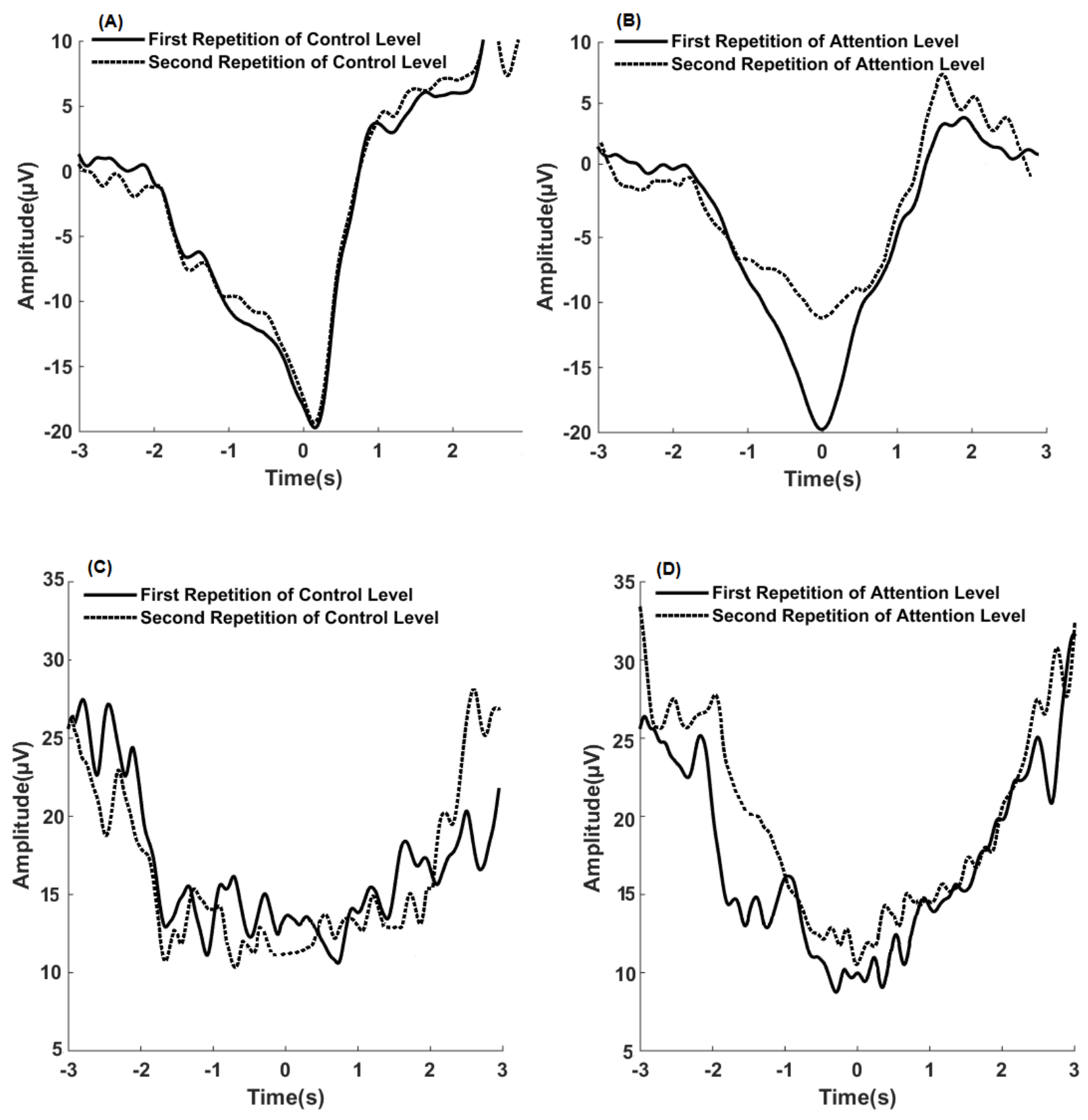

Figure 6. (a) and (b) represent grand average of MRCP of stroke patient with two levels of attention. (a) Two repetitions of movement in normal attention level while VPN is around $-19.8 \pm 12.2 \mu \mathrm{V}$ occurred nearly at $78 \mathrm{~ms}$ after movement onset. (b) Two repetitions of diverted attention level when VPN is about $-9.8 \pm 7.7 \mu \mathrm{V}$ and occurred at $103 \mathrm{~ms}$ after movement onset. (c) and (d) shows sample of variability in two repetitions within patient group in both levels of attention. (c) the variability in control level and (d) the variabity in attention level. The variability was increased in domain of [-2 -1] before TPN in attention level. TPN is the place of minimum variability. 
Table 1. Mean values and the corresponding $\mathrm{p}$ values of the single feature comparison in channel $\mathrm{Cz}$ between the two attention levels in the complex oddball tasks.

\begin{tabular}{|c|c|c|c|c|}
\hline $\begin{array}{l}\text { Temporal } \\
\text { Features }\end{array}$ & Participants & $\begin{array}{c}\text { Mean } \pm \text { SD } \\
\text { of Control } \\
\text { level }\end{array}$ & $\begin{array}{c}\text { Mean } \pm \text { SD } \\
\text { of High } \\
\text { Attention } \\
\text { Drift }\end{array}$ & $P$ value \\
\hline \multirow{2}{*}{ VPN } & Healthy & $-18.8 \pm 7.5 \mu \mathrm{V}$ & $-10.6 \pm 7.7 \mu \mathrm{V}$ & $<0.05$ \\
\hline & Patient & $-7.7 \pm 5.2 \mu \mathrm{V}$ & $-3.8 \pm 4.3 \mu \mathrm{V}$ & $<0.05$ \\
\hline \multirow[t]{2}{*}{ TPN } & Healthy & $-85.1 \pm 13.1$ & $-50.9 \pm 17.5$ & $>0.05$ \\
\hline & Patient & $93.7 \pm 12.3$ & $128.4 \pm 15.4$ & $>0.05$ \\
\hline \multirow[t]{2}{*}{ Slope 10} & Healthy & $-14.5 \pm 5.3$ & $-15.9 \pm 5.5$ & $>0.05$ \\
\hline & Patient & $-6.2 \pm 5.1$ & $-3.6 \pm 3.4$ & $<0.05$ \\
\hline \multirow[t]{2}{*}{ Slope 21} & Healthy & $-8 \pm 1.2$ & $-6.6 \pm 1.1$ & $>0.05$ \\
\hline & Patient & $-5.3 \pm 4.2$ & $-4 \pm 2.7$ & $>0.05$ \\
\hline \multirow[t]{2}{*}{ Slope 20} & Healthy & $-12.2 \pm 7.1$ & $-11.4 \pm 7.5$ & $>0.05$ \\
\hline & Patient & $-6.5 \pm .78$ & $-2.8 \pm .73$ & $<0.05$ \\
\hline \multirow[t]{2}{*}{$\mathbf{R R}$} & Healthy & $14 \pm 7.9$ & $14.1 \pm 8.6$ & $>0.05$ \\
\hline & Patient & $11.3 \pm 9.4$ & $9.3 \pm 8.1$ & $>0.05$ \\
\hline \multirow[t]{2}{*}{$\begin{array}{c}\text { Variability } \\
10\end{array}$} & Healthy & $10.2 \pm 4.1$ & $11 \pm 4.8$ & $>0.05$ \\
\hline & Patient & $8.7 \pm 12.2$ & $9.2 \pm 9.6$ & $>0.05$ \\
\hline \multirow[t]{2}{*}{$\begin{array}{c}\text { Variability } \\
21\end{array}$} & Healthy & $13.5 \pm 4.3$ & $16.9 \pm 6$ & $<0.05$ \\
\hline & Patient & $15.7 \pm 7.2$ & $20.4 \pm 8.7$ & $<0.05$ \\
\hline \multirow[t]{2}{*}{$\begin{array}{c}\text { Variability } \\
20\end{array}$} & Healthy & $12.6 \pm 5.1$ & $13.1 \pm 5.8$ & $>0.05$ \\
\hline & Patient & $17.4 \pm 8.1$ & $19.8 \pm 9.2$ & $>0.05$ \\
\hline
\end{tabular}


Table 2. Response factor of movement detection in drifted attention situations for healthy and patients and also for two levels of task demand from single channel $\mathrm{Cz}$ and its surrounding channel resulted of using large Laplacian.

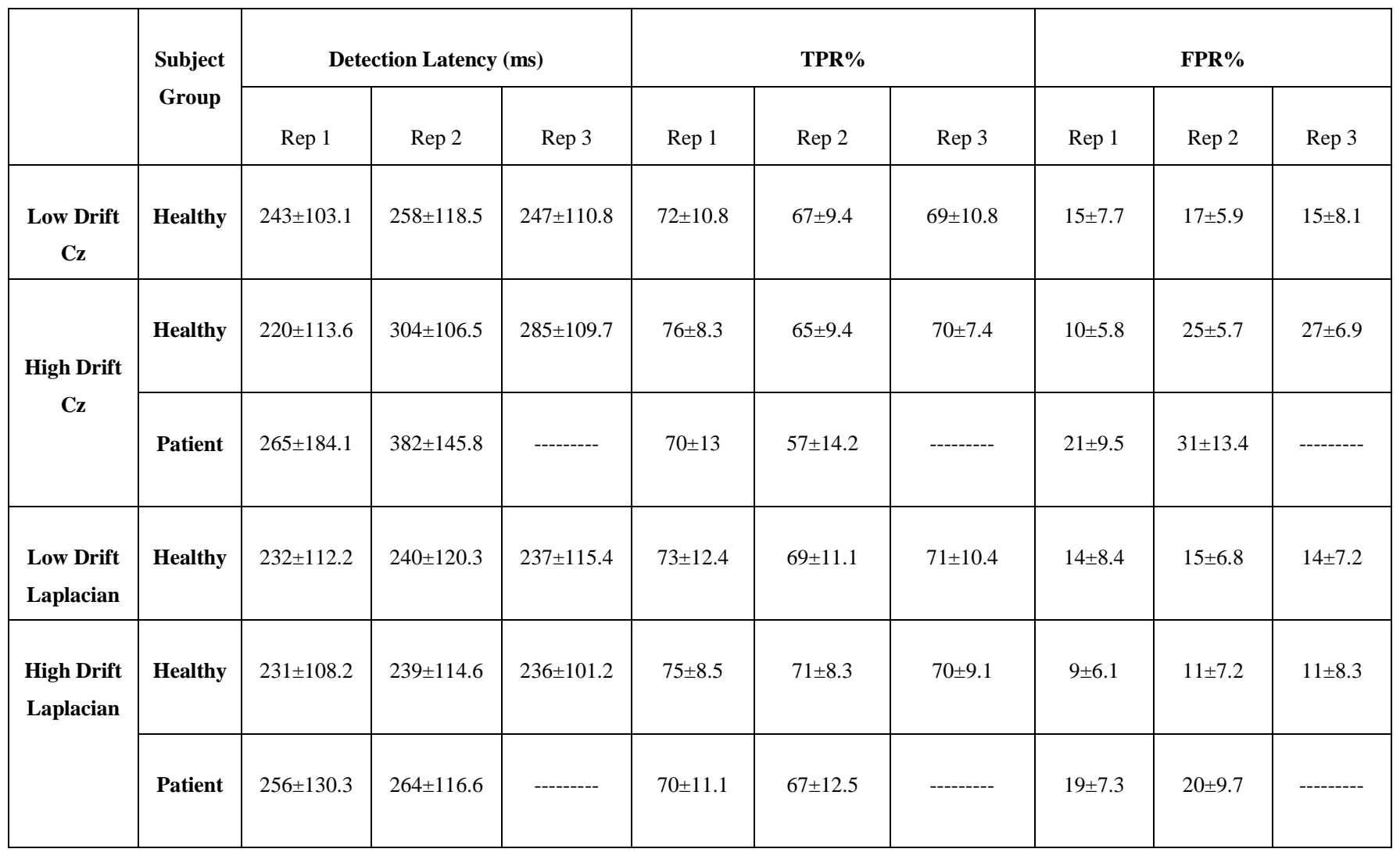


Table 3. Mean values and the corresponding $\mathrm{p}$ values of the single feature comparison in Laplacian of $\mathrm{Cz}$ between the two attention levels in the complex oddball tasks.

\begin{tabular}{|c|c|c|c|c|}
\hline $\begin{array}{l}\text { Temporal } \\
\text { Features }\end{array}$ & Participants & $\begin{array}{c}\text { Mean } \pm S D \\
\text { of Control } \\
\text { level }\end{array}$ & $\begin{array}{c}\text { Mean } \pm \text { SD } \\
\text { of High } \\
\text { Attention } \\
\text { Drift }\end{array}$ & $P$ value \\
\hline \multirow{2}{*}{ VPN } & Healthy & $-7.8 \pm 2.1 \mu \mathrm{V}$ & $-7.7 \pm 3.1 \mu \mathrm{V}$ & $>0.05$ \\
\hline & Patient & $-3.5 \pm 2.4 \mu \mathrm{V}$ & $-4 \pm 2.7 \mu \mathrm{V}$ & $>0.05$ \\
\hline \multirow{2}{*}{ TPN } & Healthy & $20.3 \pm 12$ & $50.7 \pm 20.4$ & $>0.05$ \\
\hline & Patient & $110 \pm 20.4$ & $118.6 \pm 18.7$ & $>0.05$ \\
\hline \multirow{2}{*}{ Slope 10} & Healthy & $-6.3 \pm 3.4$ & $-5.1 \pm 4.2$ & $>0.05$ \\
\hline & Patient & $-4.7 \pm 4.2$ & $-4.3 \pm 3.5$ & $>0.05$ \\
\hline \multirow{2}{*}{ Slope 21} & Healthy & $-5.3 \pm .8$ & $-4.6 \pm 1.5$ & $>0.05$ \\
\hline & Patient & $-4.2 \pm 2.8$ & $-3.8 \pm 3.2$ & $>0.05$ \\
\hline \multirow{2}{*}{ Slope 20} & Healthy & $-7.2 \pm 5.4$ & $-6.1 \pm 4.5$ & $>0.05$ \\
\hline & Patient & $-4.4 \pm 1.2$ & $-3.9 \pm .9$ & $>0.05$ \\
\hline \multirow{2}{*}{$\mathbf{R R}$} & Healthy & $8.3 \pm 4.2$ & $7.5 \pm 4.6$ & $>0.05$ \\
\hline & Patient & $6.3 \pm 5.4$ & $6.1 \pm 5.1$ & $>0.05$ \\
\hline \multirow{2}{*}{$\begin{array}{c}\text { Variability } \\
10\end{array}$} & Healthy & $8.7 \pm 3.8$ & $9 \pm 5.2$ & $>0.05$ \\
\hline & Patient & $7.5 \pm 7.2$ & $8.3 \pm 7.6$ & $>0.05$ \\
\hline \multirow{2}{*}{$\begin{array}{c}\text { Variability } \\
21\end{array}$} & Healthy & $6.2 \pm 3.8$ & $6.9 \pm 4.1$ & $>0.05$ \\
\hline & Patient & $9.7 \pm 4.2$ & $10.2 \pm 4.8$ & $>0.05$ \\
\hline \multirow{2}{*}{$\begin{array}{c}\text { Variability } \\
20\end{array}$} & Healthy & $8.4 \pm 4.5$ & $8.9 \pm 5.8$ & $>0.05$ \\
\hline & Patient & $11.1 \pm 6.3$ & $12.5 \pm 8.2$ & $>0.05$ \\
\hline
\end{tabular}

